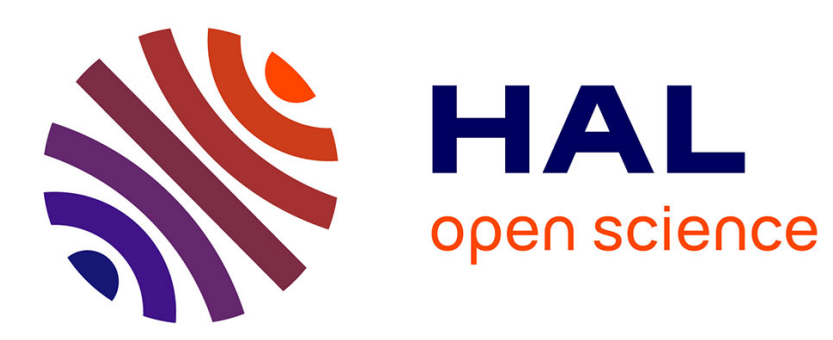

\title{
Approximate Shortest Paths Avoiding a Failed Vertex: Optimal Size Data Structures for Unweighted Graph
}

Neelesh Khanna, Surender Baswana

\section{To cite this version:}

Neelesh Khanna, Surender Baswana. Approximate Shortest Paths Avoiding a Failed Vertex: Optimal Size Data Structures for Unweighted Graph. 27th International Symposium on Theoretical Aspects of Computer Science - STACS 2010, Inria Nancy Grand Est \& Loria, Mar 2010, Nancy, France. pp.513-524. inria-00455747

\section{HAL Id: inria-00455747 https://hal.inria.fr/inria-00455747}

Submitted on 11 Feb 2010

HAL is a multi-disciplinary open access archive for the deposit and dissemination of scientific research documents, whether they are published or not. The documents may come from teaching and research institutions in France or abroad, or from public or private research centers.
L'archive ouverte pluridisciplinaire HAL, est destinée au dépôt et à la diffusion de documents scientifiques de niveau recherche, publiés ou non, émanant des établissements d'enseignement et de recherche français ou étrangers, des laboratoires publics ou privés. 


\title{
APPROXIMATE SHORTEST PATHS AVOIDING A FAILED VERTEX : OPTIMAL SIZE DATA STRUCTURES FOR UNWEIGHTED GRAPHS
}

\author{
NEELESH KHANNA ${ }^{1}$ AND SURENDER BASWANA ${ }^{2}$ \\ ${ }^{1}$ Oracle India Pvt. Ltd, Bangalore-560029, India. \\ E-mail address: neelesh.khanna@gmail.com \\ ${ }^{2}$ Indian Institute of Technology Kanpur, India. \\ E-mail address: sbaswana@cse.iitk.ac.in
}

\begin{abstract}
Let $G=(V, E)$ be any undirected graph on $V$ vertices and $E$ edges. A path $\mathbf{P}$ between any two vertices $u, v \in V$ is said to be $t$-approximate shortest path if its length is at most $t$ times the length of the shortest path between $u$ and $v$. We consider the problem of building a compact data structure for a given graph $G$ which is capable of answering the following query for any $u, v, z \in V$ and $t>1$. report $t$-approximate shortest path between $u$ and $v$ when vertex $z$ fails

We present data structures for the single source as well all-pairs versions of this problem. Our data structures guarantee optimal query time. Most impressive feature of our data structures is that their size nearly match the size of their best static counterparts.
\end{abstract}

\section{Introduction}

The shortest paths problem is a classical and well studied algorithmic problem of computer science. This problem requires processing of a given graph $G=(V, E)$ on $n=|V|$ vertices and $m=|E|$ edges to compute a data structure using which shortest path or distance between any two vertices can be efficiently reported. Two famous and thoroughly studied versions of this problem are single source shortest paths (SSSP) problem and allpairs shortest paths (APSP) problem.

Most of the applications of the shortest paths problem involve real life graphs and networks which are prone to failure of nodes (vertices) and links (edges). This has motivated researchers to design dynamic solution for the shortest paths problem. For this purpose, one has to first develop a suitable model for the shortest paths problem in dynamic networks. In fact two such models exists, and each of them has its own algorithmic objectives.

The shortest paths problem in the first model is described as follows : There is an initial graph followed by an on-line sequence of insertion and deletion of edges interspersed

1998 ACM Subject Classification: E.1 [Data Structures]:Graphs and Networks; G.2.2[Discrete Mathematics]:Graph Theory - Graph Algorithms .

Key words and phrases: Shortest path, distance, distance queries, oracle.

Part of this work was done while the authors were at Max-Planck Institute for Computer Science, Saarbruecken, Germany during the period May-July 2009.

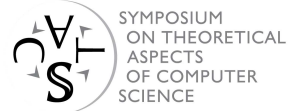


with shortest path (or distance) queries. Each query has to be answered with respect to the graph which exists at that moment (incorporating all the updates preceding the query on the initial graph). A trivial solution of this problem is to recompute all-pairs shortest paths from scratch after each update. This is certainly a wasteful approach since a single update usually does not cause a huge change in the all-pairs distance information. Therefore, the algorithmic objective here is to maintain a data structure which can answer distance query efficiently and can be updated after any edge insertion or deletion in an efficient manner. In particular, the time required to update the data structure has to be substantially less than the running time of the best static algorithm. Many novel algorithms have been designed in the last ten years for this problem and its variants (see [6] and the references therein).

On one hand the first model is important since it captures the worst possible hardness of any dynamic graph problem. On the other hand, it can also be considered as a pessimistic model for real life networks. It is true that the networks are never immune to failures. But in addition to it, it is also rare to have networks which may have arbitrary number of failures in normal circumstances. It is essential for network designers to choose suitable technology to make sure that the failures are quite infrequent in the network. In addition, when a vertex or edge fails (goes down), it does not remain failed/down indefinitely. Instead, it comes up after some finite time due to simultaneous repair mechanism going on in the network. These aspects can be captured in the second model which takes as input a graph and a number $\ell \ll n$. This model assumes that there will be at most $\ell$ vertices or edges which may be inactive at any time, though the corresponding set of failed vertices or edges may keep changing as the time progresses : the old failed vertices become active while some new active vertices may fail. The algorithmic objective in this model is to preprocess the given graph to construct a compact data structure which for any subset $S$ of at most $\ell$ vertices may answer the following query for any $u, v \in V$.

Report the shortest-path (or distance) from $u$ to $v$ in $G \backslash S$.

It is desired that each query gets answered in optimal time : retrieval of distance in $O(1)$ time and the shortest path in time which is of the order of the number of its edges. The ultimate research goal would be to understand the complexity of the above problem for any given value $\ell$. In this pursuit, the first natural step would be to efficiently solve and thoroughly understand the complexity of the problem for the case $\ell=1$, that is, the shortest paths problem avoiding any failed vertex. Interestingly, this problem appears as a sub problem in many other related problems, namely, Vickrey pricing of networks [9], most vital node of a shortest path [11], the replacement path problem [12], and shortest paths avoiding forbidden subpaths [1].

The first nontrivial and quite significant breakthrough on the all-pairs version of this problem was made by Demetrescu et al. [7]. They designed an $O\left(n^{2} \log n\right)$ space data structure, namely distance sensitivity oracle, which is capable of reporting the shortest path between any two vertices avoiding any single failed vertex. The preprocessing time of this data structure is $O\left(m n^{2}\right)$. Recently, Bernstein and Karger [4] improved the preprocessing time to $O(m n \log n)$. Though $\Theta\left(n^{2} \log n\right)$ space bound of this all-pairs distance sensitivity oracle is optimal up to logarithmic factors, it is too large for many real life graphs which appear in various large scale applications [13]. In most of these graphs usually $m \ll n^{2}$, hence a table of $\Theta\left(n^{2}\right)$ size may be too large for practical purposes. However, it is also known [7] that even a data structure which reports exact distances from a fixed source avoiding a single failed vertex will require $\Omega\left(n^{2}\right)$ space in the worst case. So approximation seems to be the only way to design a small space compact data structure for the problem of shortest 
paths avoiding a failed vertex. A path between $u, v \in V$ is said to be $t$-approximate shortest path if its length is at most $t$ times that of the shortest path between the two. The factor $t$ is usually called the stretch. We would like to state here that many algorithms and data structures have been designed in the last fifteen years for the static all-pairs approximate shortest paths (see $[2,13]$ and references therein). The prime motivation underlying these algorithms has been to achieve sub-quadratic space and/or sub-cubic preprocessing time for the static APSP problem. However, no data structure was designed in the past for approximate shortest paths avoiding any failed vertex.

In this paper, we present really compact data structures which are capable of reporting approximate shortest paths between two vertices avoiding any failed vertex in undirected graphs. The most impressive feature of our data structures is their nearly optimal size. In fact their size almost matches the size of their best static counterparts.

\subsection{New Results}

Single source approximate shortest paths avoiding any failed vertex.

First we address weighted graphs. For the weighted graphs, we present an $O(m \log n)$ time constructible data structure of size $O(n \log n)$ which can report 3-approximate shortest path from the source to any vertex $v \in V$ avoiding any $x \in V$. We then consider the case

of undirected unweighted graphs. For these graphs, we present an $O\left(n \frac{\log n}{\epsilon^{3}}\right)$ space data structure which can even report $(1+\epsilon)$-approximate shortest path for any $\epsilon>0$.

All-pairs approximate shortest paths avoiding any failed vertex.

Among the existing data structures for static all-pairs approximate shortest paths, the approximate distance oracle of Thorup and Zwick [13] stands out due to its amazing features. Thorup and Zwick [13] showed that an undirected graph can be preprocessed in sub-cubic time to build a data structure of size $O\left(k n^{1+1 / k}\right)$ for any $k>1$. This data structure, despite of its sub-quadratic size, is capable of reporting $(2 k-1)$-approximate distance between any two vertices in $O(k)$ time (and the corresponding approximate shortest path in optimal time), and hence the name oracle. Moreover, the size-stretch trade off achieved by this data structure is essentially optimal. It is a very natural question to explore whether it is possible to design all-pairs approximate distance oracle which may handle single vertex failure. We show that it is indeed possible for unweighted graphs. For this purpose, we suitably modify the approximate distance oracle of Thorup and Zwick [13] using some new insights and our single source data structure mentioned above. These modifications make the approximate shortest-paths oracle of Thorup and Zwick handle vertex failure easily, and (surprisingly) still preserving the old (optimal) trade-off between the space and the stretch. For precise details, see Theorem 5.3.

For the algorithmic details missing in this extended abstract due to page limitations, we suggest the reader to refer to the journal version [10]. Our data structures can be easily adapted for handling edge failure as well without any increase in space or time complexity.

\section{Preliminaries}

We use the following notations and definitions in the context of a given undirected graph $G=(V, E)$ with $n=|V|, m=|E|$ and a weight function $\omega: E \rightarrow \mathbf{R}^{+}$.

- $T_{r}$ : single source shortest path tree rooted at $r$.

- $\mathbf{P}(x, y)$ : the shortest path between $x$ and $y$. 
- $\delta(x, y)$ : the length of the shortest path between $x$ and $y$.

- $\mathbf{P}(x, y, z)$ : the shortest path between $x$ and $y$ avoiding vertex $z$.

- $\delta(x, y, z)$ : the length of the shortest path between $x$ and $y$ avoiding vertex $z$.

- $T_{r}(x)$ : the subtree of $T_{r}$ rooted at $x$.

- $G_{r}(x)$ : the subgraph induced by the vertices of set $T_{r}(x)$ and augmented by vertex $r$ and edges from $r$ as follows. For each $v \in T_{r}(x)$ with neighbors outside $T_{r}(x)$, keep an edge $(r, v)$ of weight $=\min _{(u, v) \in E, u \notin T_{r}(x)}(\delta(r, u)+\omega(u, v))$.

- $P:: Q$ : a path formed by concatenating path $Q$ at the end of path $P$ with an edge $(u, v) \in E$, where $u$ is the last vertex of $P$ and $v$ is the first vertex of $Q$.

- $E(X)$ : the set of edges from $E$ with at least one endpoint in $X$.

Our algorithms will also use a data structure for answering lowest common ancestor (LCA) queries on $T_{r}$. There exists an $O(n)$ time computable data structure which occupies $O(n)$ space and can answer any LCA query in $O(1)$ time (see [3] and references therein).

\section{Single source 3-approximate shortest paths avoiding a failed vertex}

We shall first solve a simpler sub-problem where the vertex which may fail belong to a given path $P \in T_{r}$. Then we use divide and conquer strategy wherein we decompose $T_{r}$ into a set of disjoint paths and for each such path, we solve this sub-problem.

\subsection{Solving the Sub-Problem : the failures of a vertex from a given path $\mathbf{P}(r, t)$}

Given the shortest path tree $T_{r}$, let $\mathbf{P}(r, t)=\left\langle r\left(=x_{0}\right), x_{1}, \ldots, x_{k}(=t)\right\rangle$ be any shortest path present in $T_{r}$. We shall design an $O(n)$ space data structure which will support retrieval of a 3 -approximate shortest path from $r$ to any $v \in V$ when some vertex from $\mathbf{P}(r, t)$ fails. The preprocessing time of our algorithm will be $O(m+n \log n)$ which matches that of Dijkstra's algorithm. The algorithm is inspired by the algorithm of Nardelli et al. [11] for computing the most vital vertex on a shortest path. Consider vertex $x_{i}$ lying on the path $\mathbf{P}(r, t)$. We

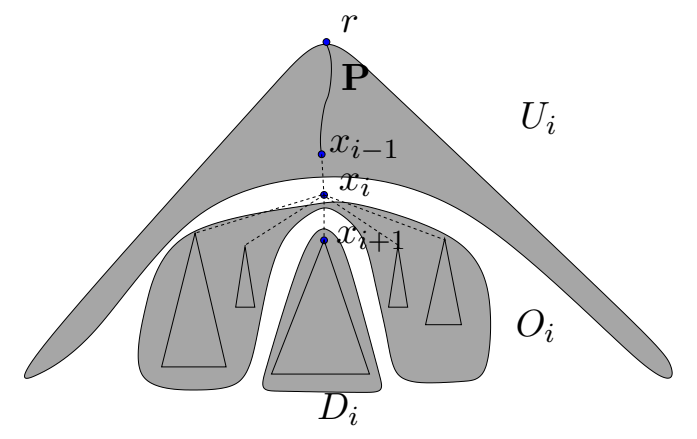

Figure 1: Partitioning of the shortest path tree $T_{r}$ at $x_{i} \in \mathbf{P}$

partition the tree $T_{r} \backslash\left\{x_{i}\right\}$ into the following 3 parts (see Figure 1).

(1) $U_{i}$ : the tree $T_{r}$ after removing the subtree $T_{r}\left(x_{i}\right)$

(2) $D_{i}:$ the subtree of $T_{r}$ rooted at $x_{i+1}$

(3) $O_{i}$ : the portion of $T_{r}$ left after removing $U_{i}, x_{i}$, and $D_{i}$.

Note that a vertex of the tree $T_{r}$ is either a vertex of the path $\mathbf{P}(r, t)$ or it belongs to some $O_{i}$ for some $i$. We build the following two data-structures of total $O(n)$ size. 
(1) a data structure to retrieve 3-approximate shortest path from $r$ to any $v \in D_{i}$.

(2) a data structure to retrieve 3-approximate shortest path from $r$ to any $v \in O_{i}$.

3.1.1. Data structure for 3-approximate shortest paths to vertices of $D_{i}$ when $x_{i}$ has failed. Consider the vertex $x_{i+1}$ and any other vertex $y \in D_{i}$. Note that the shortest path $\mathbf{P}\left(x_{i+1}, y\right)$ remains intact even after removal of $x_{i}$, and its length is certainly less than $\delta(r, y)$. Based on this simple observation one can intuitively see that in order to travel from $r$ to $y$ when $x_{i}$ fails, we may travel along shortest route to $x_{i+1}$ (that is $\mathbf{P}\left(r, x_{i+1}, x_{i}\right)$ ) and then along $\mathbf{P}\left(x_{i+1}, y\right)$. Using triangle inequality and the fact that the graph is undirected, the length of this path $\mathbf{P}\left(r, x_{i+1}, x_{i}\right):: \mathbf{P}\left(x_{i+1}, y\right)$ can be approximated as follows.

$$
\begin{aligned}
\delta\left(r, x_{i+1}, x_{i}\right)+\delta\left(x_{i+1}, y\right) & \leq \delta\left(r, y, x_{i}\right)+\delta\left(y, x_{i+1}, x_{i}\right)+\delta\left(x_{i+1}, y\right) \\
& \leq \delta\left(r, y, x_{i}\right)+2 \delta\left(x_{i+1}, y\right) \\
& \leq \delta\left(r, y, x_{i}\right)+2 \delta(r, y) \leq 3 \delta\left(r, y, x_{i}\right)
\end{aligned}
$$

Therefore, in order to support retrieval of 3-approximate shortest path to any $v \in D_{i}$ in optimal time, it suffices to store the path $\mathbf{P}\left(r, x_{i+1}, x_{i}\right)$.

In order to devise ways of efficient computation and compact storage of $\mathbf{P}\left(r, x_{i+1}, x_{i}\right)$ for a given $i$, we use the following lemma about the structure of the path $\mathbf{P}\left(r, x_{i+1}, x_{i}\right)$.

Lemma 3.1. The shortest path $\boldsymbol{P}\left(r, x_{i+1}, x_{i}\right)$ is of the form $P_{1}:: P_{2}$ where $P_{1}$ is a shortest path from $r$ in the subgraph induced by $U_{i} \cup O_{i}$, and $P_{2}$ is a path present in $D_{i}$.

It follows that in order to compute $\mathbf{P}\left(r, x_{i+1}, x_{i}\right)$, first we need to compute shortest paths from $r$ in the subgraph induced by $U_{i} \cup O_{i}$. Let $\delta_{i}(r, v)$ denote the distance from $r$ to $v \in$ $U_{i} \cup O_{i}$ in this subgraph. Note that $\delta_{i}(r, v)$ for $v \in U_{i}$ and the corresponding shortest path is the same as in the original graph, and is already present in $T_{r}$. For computing shortest paths from $r$ to vertices of $O_{i}$, we build a shortest path tree (denoted as $T_{r}\left(O_{i}\right)$ ) from $r$ in the subgraph induced by vertices $O_{i} \cup\{r\}$ and the following additional edges. For each $z \in O_{i}$ with at least one neighbor in $U_{i}$, we add an edge $(r, z)$ with weight $=\min _{(u, z) \in E, u \in U_{i}}(\delta(r, u)+$ $\omega(u, z))$. Applying Lemma 3.1 , let $\left(y_{i}, z_{i}\right)$ be the edge of $\mathbf{P}\left(r, x_{i+1}, x_{i}\right)$ joining the sub path present in $U_{i} \cup O_{i}$ with the sub path present in $D_{i}$. This edge can be identified using the fact that this is the edge which minimizes $\delta_{i}(r, y)+\omega(y, z)+\delta\left(x_{i+1}, z\right)$ over all $z \in D_{i}, y \in U_{i} \cup O_{i},(y, z) \in E$. The vertex $x_{i+1}$ stores the path $\mathbf{P}\left(r, x_{i+1}, x_{i}\right)$ implicitly by keeping the edge $\left(y_{i}, z_{i}\right)$ and the tree $T_{r}\left(O_{i}\right)$. The shortest path $\mathbf{P}\left(r, x_{i+1}, x_{i}\right)$ can be retrieved in optimal time using the trees $T_{r}, T_{r}\left(O_{i}\right)$, and the edge $\left(y_{i+1}, z_{i+1}\right)$. Due to mutual disjointness of $O_{i}$ 's, the overall space requirement of the data structure for retrieving $\mathbf{P}\left(r, x_{i+1}, x_{i}\right)$ for all $i \leq k$ will be $O(n)$.

3.1.2. Data structure for 3-approximate shortest paths to vertices of $O_{i}$ when $x_{i}$ has failed. In order to compute 3-approximate shortest path to $O_{i}$ upon failure of $x_{i}$, we shall use the approximate shortest paths to $D_{i}$ as computed above. Here we use an interesting observation which states that if we have a data structure to retrieve $\alpha$-approximate shortest paths from $r$ to vertices of $D_{i}$ when $x_{i}$ fails, then we can use it to have a data-structure to retrieve $\alpha$-approximate shortest paths to vertices of $O_{i}$ as well. To prove this result, this is how we proceed. Consider the subgraph induced by $O_{i}$ and augmented with vertex $r$ and some extra edges which are defined as follows. 
- For each $o \in O_{i}$ having neighbors from $U_{i}$, keep an edge $(r, o)$ and assign it weight $=\min _{(u, o) \in E, u \in U_{i}}(\delta(r, u)+\omega(u, o))$.

- For each $o \in O_{i}$ having neighbors from $D_{i}$, keep an edge $(r, o)$ and assign it weight $=$ $\min _{(u, o) \in E, u \in D_{i}}\left(\hat{\delta}\left(r, u, x_{i}\right)+\omega(u, o)\right)$, where $\hat{\delta}\left(r, u, x_{i}\right)$ is the $\alpha$-approximate distance to $u$ upon failure of $x_{i}$. (In the present situation we have $\alpha=3$.)

Let us denote this graph as $G_{r}\left(O_{i}\right)$. Observation 3.3 is based on the following lemma which is easy to prove.

Lemma 3.2. The Dijkstra's algorithm from $r$ in the graph $G_{r}\left(O_{i}\right)$ computes $\alpha$-approximate shortest paths from $r$ to all $v \in O_{i}$ avoiding $x_{i}$.

Observation 3.3. If we can design a data structure for retrieving $(1+\epsilon)$-approximate shortest paths from $r$ to vertices of $D_{i}$ upon failure of $x_{i}$, then it can also be used to design a data structure which can support retrieval of $(1+\epsilon)$-approximate shortest paths to all vertices of the graph upon failure of $x_{i}$.

We compute and store the shortest path tree rooted at $r$ in the graph $G_{r}\left(O_{i}\right)$. This tree along with the tree $T_{r}$ and the data structure described in the previous sub-section suffice for retrieval of 3-approximate shortest paths to $o \in O_{i}$ upon failure of $x_{i}$.

Query answering: Suppose the oracle receives a query asking for approximate shortest path from $r$ to $v$ avoiding $x_{i} \in \mathbf{P}(r, t)$. It first invokes lowest common ancestor (LCA) query between $v$ and $x_{i}$ on $T_{r}$. If $L C A\left(v, x_{i}\right) \neq x_{i}$, the shortest path from $r$ to $v$ remains unaffected and so it reports the path $\mathbf{P}(r, t)$. Otherwise, it determines if $v \in D_{i}$ or $v \in O_{i}$. Depending upon the two cases, it reports the approximate shortest path between $r$ and $v_{i}$ using one of the two data structures described above.

Theorem 3.4. An undirected weighted graph $G=(V, E)$, a source $r \in V$, and a shortest path $P \in T_{r}$ can be processed in $O(m+n \log n)$ time to build a data structure of $O(n)$ space which can report 3-approximate shortest path from $r$ to any $v \in V$ avoiding any single failed vertex from $P$.

\subsection{Handling the failure of any vertex in $T_{r}$}

We follow divide and conquer strategy based on the following simple lemma.

Lemma 3.5. There exists an $O(n)$ time algorithm to compute a path $P$ in $T_{r}$ whose removal splits $T_{r}$ into a collection of disjoint subtrees $T_{r}\left(v_{1}\right), \ldots T_{r}\left(v_{j}\right)$ such that

- $\left|T_{r}\left(v_{i}\right)\right|<n / 2$ for each $i \leq j$.

- $P \cup_{i} T_{r}\left(v_{i}\right)=T$ and $P \cap T_{r}\left(v_{i}\right)=\emptyset \forall i$.

First we compute the path $P \in T_{r}$ as mentioned in Lemma 3.5. We build the data structure for handling failure of any vertex from $P$ by executing the algorithm of Theorem 3.4. Let $v_{1}, \ldots, v_{j}$ be the roots of the sub trees of $T_{r}$ connected to the path $P$ with an edge. For each $1 \leq i \leq j$, we solve the problem recursively on the subgraph $G_{r}\left(v_{i}\right)$, and build the corresponding data structures. Lemma 3.5 and Theorem 3.4 can be used in straight forward manner to prove the following theorem.

Theorem 3.6. An undirected weighted graph $G=(V, E)$ can be processed in $O(m \log n+$ $\left.n \log ^{2} n\right)$ time to build a data structure of size $O(n \log n)$ which can answer, in optimal time, any 3-approximate shortest path query from a given source $r$ to any vertex $v \in V$ avoiding any single failed vertex. 


\section{Single source $(1+\epsilon)$-approximate shortest paths avoiding a failed vertex}

In this section, we shall present a compact data structure for single source $(1+\epsilon)$ approximate shortest paths avoiding a failed vertex in an unweighted graph. Let level $(v)$ denote the level (or distance from $r$ ) of vertex $v$ in the tree $T_{r}$. Let $U_{x}, D_{x}, O_{x}$ denote the partitions of the tree $T_{r}$ formed by deletion of vertex $x$, with the same meaning as that of $U_{i}, D_{i}, O_{i}$ defined for $x_{i}$ in the previous section. On the basis of Observation 3.3, our objective is to build a compact data structure which will support retrieval of $(1+\epsilon)$ approximate shortest-paths to vertices of $D_{x}$ upon failure of $x$ for any $x \in V$. Let uchild $(x)$ denote the root of the subtree corresponding to $D_{x}$ (it is similar to $x_{i+1}$ in case of $D_{i}$ ). For reporting approximate distance between $r$ and $v \in D_{x}$ when $x$ fails, the data structure of previous section reports path of length $\delta(r$, uchild $(x), x)+\delta(\operatorname{uchild}(x), v)$ which is bounded by $\delta(r, v, x)+2 \delta$ uchild $(x), v)$. It should be noted that the approximation factor associated with it is already bounded by $(1+\epsilon)$ for any $\epsilon>0$ if the following condition holds.

C : $\operatorname{uchild}(x)$ is close to $v$, that is, $\delta(\operatorname{uchild}(x), v) \leq \frac{\epsilon}{2} \delta(r, v)$.

We shall build a supplementary data structure which will ensure that whenever the condition $\mathbf{C}$ does not hold, there will be some ancestor $w$ of $v$ lying on $\mathbf{P}(x, v)$, called a special vertex, satisfying the following two properties.

(1) $\delta(w, v) \ll \delta(r, v)$, that is $w$ is much closer to $v$ than $r$.

(2) vertex $w$ stores approximate shortest path to $r$ avoiding $x$ (with the approximation factor arbitrarily close to 1 ).

We shall refer to such vertices $w$ as special-vertices.

\subsection{Constructing the set of special vertices}

Let $h$ be the height of BFS tree rooted at $r$. Let $L$ be a set of integers such that $L=\left\{i \mid\left\lfloor(1+\epsilon)^{i}\right\rfloor<h\right\}$. For a given $i \in L$, we define a subset $S_{i}$ of special vertices as $S_{i}=\left\{u \in V \mid\right.$ level $\left.(u)=\left\lfloor(1+\epsilon)^{i}\right\rfloor \wedge\left|T_{r}(u)\right| \geq \operatorname{elevel}(u)\right\}$. We define the set of special vertices as $S=\cup_{\forall i \in L} S_{i}$. In addition, we also introduce the following terminologies.

- $S(v)$ : the nearest ancestor of $v$ which belongs to set $S$.

- $V(u)$ : For a vertex $u \in S, V(u)$ denotes the set of vertices $v \in V$ with $S(v)=u$. In essence, the vertex $u$ will serve as the special vertex for each vertex from $V(u)$. For failure of any vertex $x \in \mathbf{P}(r, u)$, each vertex of set $V(u)$ will query the data structure stored at $u$ for retrieval of approximate shortest path/distance from the source.

We now state two simple lemmas based on the above construction.

Lemma 4.1. Let $v \in V \backslash S$, then $\delta(v, S(v)) \leq\left(\frac{2 \epsilon}{1+\epsilon}\right) \operatorname{level}(v)$ if $\epsilon<1$

Lemma 4.2. Let $u$ be a vertex at level $\ell$ and $u \in S$. Then $V(u) \geq \epsilon \ell$.

If we can ensure that the data structure for a special vertex $u$ (for retrieving approximate shortest paths from $r$ upon failure of any $x \in \mathbf{P}(r, u))$ is of size $O($ level $(u))$, then it would follow from Lemma 4.2 that the space required by our supplementary data structure will be linear in $n$. 


\subsection{The data structure for a special vertex}

Consider a special vertex $v$ with level $(v)=\left\lfloor(1+\epsilon)^{i}\right\rfloor$ We shall now describe a compact data structure stored at $v$ which will facilitate retrieval of approximate shortest path from $r$ to $v$ upon failure of any vertex $x \in \mathbf{P}(r, v)$.

Let $v^{\prime}$ be the special vertex which is present at level $\left\lfloor(1+\epsilon)^{i-1}\right\rfloor$ and is ancestor of $v$. The data structure stored at $v$ will be defined in a way that will prevent it from storing information that is already present in the data structure of some special vertex lying on $\mathbf{P}\left(r, v^{\prime}\right)$.

If $x \in \mathbf{P}\left(v^{\prime}, v\right)$, then the data structure described in the previous section itself stores a path which is $(1+2 \epsilon)$-approximation of $\mathbf{P}(r, v, x)$.

Let us now consider the nontrivial case when $x \in \mathbf{P}\left(r, v^{\prime}\right), x \neq v^{\prime}$. In order to discuss this case, we would like to introduce the notion of detour. To understand it, let us visualize the paths $\mathbf{P}(r, v, x)$ and $\mathbf{P}(r, v)$ simultaneously. Since $\mathbf{P}(r, v, x)$ and $\mathbf{P}(r, v)$ have the same end-points and $x$ doesn't lie on $\mathbf{P}(r, v, x)$, there must be a middle portion of $\mathbf{P}(r, v, x)$ which intersects $\mathbf{P}(r, v)$ at exactly two vertices, and the remaining portion of $\mathbf{P}(r, v, x)$ overlaps with $\mathbf{P}(r, v)$. This middle portion is called a detour. We now define it more formally. Let $a$ and $b$ be two vertices on the shortest path $\mathbf{P}(r, v)$. We use $a \prec b$ to denote that vertex $a$ is closer to $r$ than vertex $b$. The notation $a \preceq b$ would mean that either $a \prec b$ or $a=b$. . So here is the definition of detour (and the underlying observation).

Definition 4.3. Let $x \in \mathbf{P}(r, y)$. When $x$ fails, the path $\mathbf{P}(r, y, x)$ will be of the form of $\mathbf{P}(r, a):: p_{a, b}:: \mathbf{P}(b, y)$, where $r \preceq a \prec x \prec b \preceq y$ and the path $p_{a, b}$ is such that $p_{a, b} \cap \mathbf{P}(a, b)=\{a, b\}$. In other words, $p_{a, b}$ meets $\mathbf{P}(a, b)$ only at the end points. We shall call $p_{a, b}$ as the detour associated with the shortest path $\mathbf{P}(r, y, x)$.

Let $p_{a, b}$ represent the detour w.r.t. to $\mathbf{P}(r, v, x)$. The handling of failure of vertices $x \in \mathbf{P}(r, v)$ which lie above $v^{\prime}$ would depend upon the detour $p_{a, b}$. This detour can be of any of the following types (see Figure 2 for illustration).

- I : $b \preceq v^{\prime}$.

- II : $v^{\prime} \prec b$.

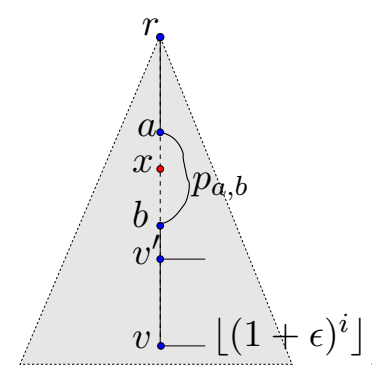

(i)

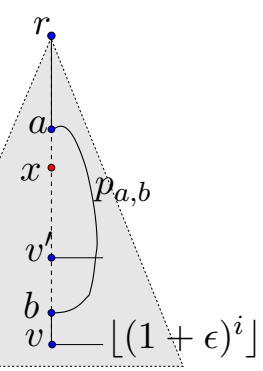

(ii)

Figure 2: $p_{a, b}$ is shortest detour of $\mathbf{P}(r, v, x)$. (i) : detour of type I, (ii) : detour of type II

Handling detours of type I is relatively easy. Let $w$ be the farthest ancestor of $v$ such that $w \in S$ and level of $w$ is greater or equal to the level of $b$. In this case, $v$ stores the corresponding detour implicitly by just keeping a pointer to the vertex $w$.

Handling detours of type II is slightly tricky since we can't afford to store each of them explicitly. However, we shall employ the following observation associated with the detours of type II to guarantee low space requirement. 
Observation 4.4. Let $\alpha_{1}, \alpha_{2}, \ldots, \alpha_{t}$ be the vertices on $\mathbf{P}(r, v)$ (in the increasing order of their levels) such that the shortest detour corresponding to $\mathbf{P}\left(r, v, \alpha_{i}\right)$ is of type II $\forall i$, then

$$
\delta\left(r, v, \alpha_{1}\right) \geq \delta\left(r, v, \alpha_{2}\right) \geq \cdots \geq \delta\left(r, v, \alpha_{t}\right)
$$

It follows from the above observation that if $\delta\left(r, v, \alpha_{i}\right) \leq(1+\epsilon) \delta\left(r, v, \alpha_{j}\right)$ for any $i<j$, then $\mathbf{P}\left(r, v, \alpha_{i}\right)$ may as well serve as $(1+\epsilon)$-approximate shortest path from $r$ to $v$ avoiding $\alpha_{j}$. In other words, we need not store the detour associated with $\mathbf{P}\left(r, v, \alpha_{j}\right)$ in such situation. Using this observation, we shall have to explicitly store only $O\left(\log _{1+\epsilon} n\right)$ detours of type II. Moreover, we do not store explicitly detours of type II whose length is much larger than level $(v)$. Specifically, if $\mathbf{P}(r, v, x) \geq \frac{1}{\epsilon} \operatorname{level}(v)$, then $v$ will merely store pointer to the path $\mathbf{P}(r, \operatorname{uchild}(x), x):: \mathbf{P}(\operatorname{uchild}(x), v)$. This ensures that each detour of type II which $v$ has to explicitly store will have length $O\left(\frac{1}{\epsilon} l e v e l(v)\right)$.

It follows from the above description that for a special vertex $v$ and $x \in \mathbf{P}(r, v)$, the data structure associated with $v$ stores $(1+2 \epsilon)$-approximation of the path $\mathbf{P}(r, v, x)$. Moreover, the total space required by the data structure associated with all the special vertices will be $O\left(n \frac{\log n}{\epsilon^{3}}\right)$. This supplementary data structure combined with the data structure of previous section can report $(1+6 \epsilon)$-approximation of $\mathbf{P}(r, z, x)$ for any $z, x \in V$.

Theorem 4.5. Given an undirected unweighted graph $G=(V, E)$, source $r \in V$, and any $\epsilon>0$, we can build a data structure of size $O\left(n \frac{\log n}{\epsilon^{3}}\right)$ that can report $(1+\epsilon)$-approximate shortest path from $r$ to any $z \in V$ avoiding any failed vertex in optimal time.

\section{All-pairs $(2 k-1)(1+\epsilon)$-approx. distance oracle avoiding a failed vertex}

We start with a brief description of the approximate distance oracle of Thorup and Zwick [13]. The key idea to achieve sub-quadratic space is to store distance from each vertex to only a small set of vertices. For retrieving approximate distance between any two vertices $u, v \in V$, it is ensured that there is a third vertex $w$ which is close to both of them, and whose distance from both of them is known. To realize this idea, Thorup and Zwick [13] introduced two novel structures called ball and cluster which are defined for any two subsets $A, B$ of vertices as follows. (here $\delta(v, B)$ denotes the distance between $v$ and its nearest vertex from $B$ ).

$$
\operatorname{Ball}(v, A, B)=\{w \in A \mid \delta(v, w)<\delta(v, B)\} \quad C(w, A, B)=\{v \in V \mid \delta(v, w)<\delta(v, B)\}
$$

Construction of $(2 k-1)$-approximate distance oracle of Thorup and Zwick [13] employs a $k$-level hierarchy $\mathbf{A}_{k}=\left\langle A_{0} \supseteq A_{1} \supseteq A_{2} \ldots \supseteq A_{k-1} \supset A_{k}\right\rangle$ of subsets of vertices as follows.

$A_{0}=V, A_{k}=\emptyset$, and $A_{i+1}$ for any $i<k-1$ is formed by selecting each vertex from $A_{i}$ independently with probability $n^{-1 / k}$.

The data structure associated with the $(2 k-1)$-approximate distance oracle of Thorup and Zwick [13] stores for each vertex $v \in V$ the following information :

- the vertices of set $\cup_{i<k} \operatorname{Ball}\left(v, A_{i}, A_{i+1}\right)$ (and their distances).

- the vertex from $A_{i}$ nearest to $v$ (to be denoted as $p_{i}(v)$ ).

Due to randomization underlying the construction of $\mathbf{A}_{k}$, the expected size of $\operatorname{Ball}\left(v, A_{i}, A_{i+1}\right)$ is $O\left(n^{1 / k}\right)$, and hence the space required by the oracle is $O\left(k n^{1+1 / k}\right)$. We shall now outline the ideas in extending the $(2 k-1)$-approximate distance oracle to handle single vertex failure. Kindly refer to the extended version [10] of this paper for complete details. 


\subsection{Overview of all-pairs approx. distance oracles avoiding a failed vertex}

Firstly the notations used by the static approximate distance oracle of [13], in particular ball and cluster, get extended for single vertex failure in a natural manner as follows. (here $\delta(v, B, x)$ is the distance between $v$ and its nearest vertex from $B$ in $G \backslash\{x\})$.

$$
\begin{gathered}
\operatorname{Ball}^{x}(v, A, B)=\{w \in A \mid \delta(v, w, x)<\delta(v, B, x)\} \\
C^{x}(w, A, B)=\{v \in V \mid \delta(v, w, x)<\delta(v, B, x)\}
\end{gathered}
$$

Let $p_{i}^{x}(v)$ denote the vertex from $A_{i}$ which is nearest to $v$ in $G \backslash\{x\}$. Along the lines of the static approximate distance oracle of Thorup and Zwick [13], the basic operation which the approximate distance oracle avoiding a failed vertex should support is the following :

Report distance (exact or approximate) between $v$ and $w \in A_{i}$ if $w \in \operatorname{Ball}^{x}\left(v, A_{i}, A_{i+1}\right)$ for any given $v, x \in V$.

However, it can be observed that we would have to support this operation implicitly instead of explicitly keeping $\operatorname{Ball}^{x}\left(v, A_{i}, A_{i+1}\right)$ for each $v, x, i$. Our starting point is the simple observation that clusters and balls are inverses of each others, that is, $w \in \operatorname{Ball}^{x}\left(v, A_{i}, A_{i+1}\right)$ is equivalent to $v \in C^{x}\left(w, A_{i}, A_{i+1}\right)$. Now we make an important observation. Consider the subgraph $\mathbf{G}_{i}(w)$ induced by the vertices of set $\cup_{x \in V} C^{x}\left(w, A_{i}, A_{i+1}\right)$. This subgraph preserves the path $\mathbf{P}(w, v, x)$ for each $x, v \in V$ if $w \in \operatorname{Ball}^{x}\left(v, A_{i}, A_{i+1}\right)$. So it suffices to keep a single source (approximate) shortest paths oracle on $\mathbf{G}_{i}(w)$ with $w$ as the root. Keeping this data structure for each $w \in A_{i}$ provides an implicit compact data structure for supporting the basic operation mentioned above. Using Theorem 4.5, it can be seen that the space required at a level $i$ will be of the order of $\sum_{w \in A_{i}}\left|\cup_{x \in V} C^{x}\left(w, A_{i}, A_{i+1}\right)\right|$, but it is not clear whether we can get an upper bound of the order of $n^{1+1 / k}$ on this quantity. Here, as a new tool, we introduce the notion of $\epsilon$-truncated balls and clusters.

Definition 5.1. Given a vertex $x$, any subsets $A, B$, and $\epsilon>0$

$$
\operatorname{Ball}^{x}(v, A, B, \epsilon)=\left\{w \in A \mid \delta(v, w, x)<\frac{\delta(v, B, x)}{1+\epsilon}\right\}
$$

Instead of dealing with the usual balls (and clusters) under deletion of single vertex, we deal with $\epsilon$-truncated balls (and clusters) under deletion of single vertex. We note that the inverse relationship between clusters and balls gets seamlessly extended to $\epsilon$-truncated balls and clusters under single vertex failure as well. That is,

$$
\sum_{w \in A_{i}}\left|\cup_{x \in V} C^{x}\left(w, A_{i}, A_{i+1}, \epsilon\right)\right|=\sum_{v \in V}\left|\cup_{x \in V} \operatorname{Ball}^{x}\left(v, A_{i}, A_{i+1}, \epsilon\right)\right|
$$

So it suffices to get an upper bound on the size of the set $\cup_{x \in V} \operatorname{Ball}^{x}\left(v, A_{i}, A_{i+1}, \epsilon\right)$ for any vertex $v \in V$. The following lemma states a very crucial property of $\epsilon$-truncated balls which leads to prove the existence of a small set $S$ of $O\left(\frac{1}{\epsilon^{2}} \log n\right)$ vertices such that

$$
\cup_{x \in V} \operatorname{Ball}^{x}\left(v, A_{i}, A_{i+1}, \epsilon\right) \subseteq \cup_{x \in S} \operatorname{Ball}^{x}\left(v, A_{i}, A_{i+1}\right) \cup \operatorname{Ball}\left(v, A_{i}, A_{i+1}\right)
$$

Lemma 5.2. In a given graph $G=(V, E)$, let $v$ be any vertex and let $u=p_{i+1}(v)$. Let $x_{1}$ and $x_{2}$ be any two vertices on the $\boldsymbol{P}(v, u)$ path with $x_{1}$ appearing closer to $v$ on this path and $\delta\left(v, A_{i+1}, x_{1}\right) \leq(1+\epsilon) \delta\left(v, A_{i+1}, x_{2}\right)$. Then

$$
\operatorname{Ball}^{x_{1}}\left(v, A_{i}, A_{i+1}, \epsilon\right) \subseteq \operatorname{Ball}\left(v, A_{i}, A_{i+1}\right) \cup \operatorname{Ball}^{x_{2}}\left(v, A_{i}, A_{i+1}\right)
$$


Proof. Let $w$ be any vertex in $A_{i}$. It suffices to show the following. If $w$ does not belong to $\operatorname{Ball}\left(v, A_{i}, A_{i+1}\right) \cup \operatorname{Ball}^{x_{2}}\left(v, A_{i}, A_{i+1}\right)$, then $w$ does not belong to $\operatorname{Ball}^{x_{1}}\left(v, A_{i}, A_{i+1}, \epsilon\right)$. The proof is based on the analysis of the following two cases.

Case 1 : The vertex $x_{2}$ is present in $\mathbf{P}\left(v, w, x_{1}\right)$.

Since, $w \notin \operatorname{Ball}\left(v, A_{i}, A_{i+1}\right)$, therefore, $\delta(v, w)$ is at least $\delta(v, u)$. Hence using triangle inequality, $\delta\left(v, x_{2}\right)+\delta\left(x_{2}, w\right) \geq \delta(v, u)$. Now $\delta(v, u)=\delta\left(v, x_{2}\right)+\delta\left(x_{2}, u\right)$ (since $x_{2}$ lies on $P(v, u))$. Hence $\delta\left(x_{2}, w\right) \geq \delta\left(x_{2}, u\right)$. Moreover, since $x_{1}$ does not appear on $\mathbf{P}\left(x_{2}, u\right)$, so $\delta\left(x_{2}, u\right)=\delta\left(x_{2}, u, x_{1}\right)$. So

$$
\delta\left(x_{2}, w, x_{1}\right) \geq \delta\left(x_{2}, u, x_{1}\right)
$$

Now it is given that $x_{2} \in \mathbf{P}\left(v, w, x_{1}\right)$, so $\mathbf{P}\left(v, w, x_{1}\right)$ must be of the form $\mathbf{P}\left(v, x_{2}, x_{1}\right)::$ $\mathbf{P}\left(x_{2}, w, x_{1}\right)$, the length of which is at least $\delta\left(v, x_{2}, x_{1}\right)+\delta\left(x_{2}, u, x_{1}\right)$ using Equation 5.2. The latter quantity is at least $\delta\left(v, u, x_{1}\right)$ which by definition is at least $\delta\left(v, A_{i+1}, x_{1}\right)$. Hence $w \notin \operatorname{Ball}^{x_{1}}\left(v, A_{i}, A_{i+1}\right)$, and therefore, $w \notin \operatorname{Ball}^{x_{1}}\left(v, A_{i}, A_{i+1}, \epsilon\right)$.

Case 2 : The vertex $x_{2}$ is not present in $\mathbf{P}\left(v, w, x_{1}\right)$.

In this case, $\delta\left(v, w, x_{1}\right)=\delta\left(v, w,\left\{x_{1}, x_{2}\right\}\right) \geq \delta\left(v, w, x_{2}\right)$. The value $\delta\left(v, w, x_{2}\right)$ is in turn at least $\delta\left(v, A_{i+1}, x_{2}\right)$ since $w \notin \operatorname{Ball}^{x_{2}}\left(v, A_{i}, A_{i+1}\right)$. It is given that $\delta\left(v, A_{i+1}, x_{2}\right) \geq$ $\frac{\delta\left(v, A_{i+1}, x_{1}\right)}{1+\epsilon}$, hence conclude that $\delta\left(v, w, x_{1}\right) \geq \frac{\delta\left(v, A_{i+1}, x_{1}\right)}{1+\epsilon}$. So $w \notin \operatorname{Ball}^{x_{1}}\left(v, A_{i}, A_{i+1}, \epsilon\right)$.

We shall now outline the construction of a small set $S$ of vertices which will satisfy Equation 5.1. Let $u=p_{i+1}(v)$ and let $\mathbf{P}(v, u)=v\left(=x_{0}\right), x_{1}, \ldots, x_{\ell}(=u)$. Observe that $\cup_{x \in V} \operatorname{Ball}^{x}\left(v, A_{i}, A_{i+1}, \epsilon\right)=\cup_{1 \leq j \leq \ell} \operatorname{Ball}^{x_{j}}\left(v, A_{i}, A_{i+1}, \epsilon\right)$. For any node $x \in \mathbf{P}(u, v)$, let $\operatorname{value}(x)=\delta\left(v, A_{i+1}, x\right)$, and let $h$ be the maximum value of any node on this path. The set $S$ is initially empty.

Let $\alpha(1)$ be the largest index from $[1, \ell]$ such that value $\left(x_{i}\right) \geq h /(1+\epsilon)$. It can be seen that for all $j<\alpha(1), \delta\left(v, A_{i+1}, x_{j}\right) \leq(1+\epsilon) \delta\left(v, A_{i+1}, x_{\alpha(1)}\right)$. Therefore, it follows from Lemma 5.2 that for each vertex $x \in\left\{x_{1}, \ldots, x_{\alpha(1)}\right\}, \operatorname{Ball}^{x}\left(v, A_{i}, A_{i+1}, \epsilon\right) \subseteq$ $\operatorname{Ball}^{x_{\alpha(1)}}\left(v, A_{i}, A_{i+1}\right) \cup \operatorname{Ball}\left(v, A_{i}, A_{i+1}\right)$. So we insert $x_{\alpha(1)}$ to $S$. Similarly $\alpha(2) \in$ $[\alpha(1)+1, \ell]$ be the greatest integer such that value $\left(x_{\alpha(2)}\right) \geq h /(1+\epsilon)^{2}$. We add $x_{\alpha(2)}$ to $S$, and so on. It can be seen that the set $S$ constructed in this manner will satisfy Equation 5.1 and its size will be $O\left(\log _{1+\epsilon} h\right)=O\left(\frac{\log n}{\epsilon}\right)$.

It can be shown using elementary probability theory that for each $x \in V$, the set $\operatorname{Ball}^{x}\left(v, A_{i}, A_{i+1}\right)$ has size $O\left(n^{1 / k} \log n\right)$ with high probability. Therefore, the construction of the set $S$ outlined above implies the following crucial bound for each $v \in V, i<k-1$ which helps us design all-pairs approximate distance oracle avoiding a failed vertex.

$$
\left|\cup_{x \in V} \operatorname{Ball}^{x}\left(v, A_{i}, A_{i+1}, \epsilon\right)\right|=O\left(n^{1 / k} \frac{\log ^{2} n}{\epsilon}\right)
$$

Using this equation, and owing to inverse relationship between clusters and balls, it follows that $\sum_{w \in A_{i}}\left|\cup_{x \in V} C^{x}\left(w, A_{i}, A_{i+1}, \epsilon\right)\right|=O\left(n^{1+1 / k} \frac{\log ^{2} n}{\epsilon}\right)$. Our all-pairs approximate distance oracle avoiding any failed vertex will keep the following data structures.

- Let $p_{i}^{x}(v, \epsilon)$ denote a vertex $w$ from $A_{i}$ with $\delta(v, w, x) \leq(1+\epsilon) \delta\left(v, p_{i}^{x}(v), x\right)$. We keep a data structure $\mathbf{N}_{i} \forall i<k$, using which we can retrieve $p_{i}^{x}(v, \epsilon)$. This data-structure is obtained by suitable augmentation of our single source $(1+\epsilon)$-approximate oracle.

- For each $w \in A_{i}$, we keep our single source $(1+\epsilon)$-approximate oracle in $\mathbf{G}_{i}(w, \epsilon)$ which is the subgraph induced by $\cup_{x \in V} C^{x}\left(w, A_{i}, A_{i+1}, \epsilon\right)$. 
It follows that the overall space required by the data structure will be $O\left(k n^{1+1 / k} \frac{\log ^{3} n}{\epsilon^{4}}\right)$. The query algorithm and the analysis on the stretch of the approximate distance reported by the oracle are similar in spirit to that of Thorup and Zwick [13] (see [10] for details).

Theorem 5.3. Given an integer $k>1$ and a fraction $\epsilon>0$, an unweighted graph $G=$ $(V, E)$ can be processed to construct a data structure which can answer $(2 k-1)(1+\epsilon)$ approximate distance query between any two nodes $u \in V$ and $v \in V$ avoiding any single failed vertex in $O(k)$ time. The total size of the data structure is $O\left(k n^{1+1 / k} \frac{\log ^{3} n}{\epsilon^{4}}\right)$.

Future work. (i) Can we design a data structure for single source $(1+\epsilon)$-approx. shortest paths avoiding a failed vertex for weighted graphs? Such a data structure will immediately extend our all-pairs approx. distance oracle avoiding a failed vertex to weighted graphs.

(ii) How to design approx. distance oracles avoiding two or more failed vertices ? Recent work of Duan and Pettie [8], and Chechik et al. [5] provides additional motivation for this.

\section{References}

[1] M. Ahmed and A. Lubiw. Shortest paths avoiding forbidden subpaths. In STACS '09: Proceedings of 26th International Symposium on Theoretical Aspects of Computer Science, pages 63-74, Freiburg, Germany, 2009. Schloss Dagstuhl - Leibniz-Zentrum fuer Informatik, Germany.

[2] S. Baswana and T. Kavitha. Faster algorithms for approximate distance oracles and all-pairs small stretch paths. In FOCS '06: Proceedings of the 47th Annual IEEE Symposium on Foundations of Computer Science, pages 591-602, Washington, DC, USA, 2006. IEEE Computer Society.

[3] M. A. Bender and M. Farach-Colton. The lca problem revisited. In LATIN '00: Proceedings of the 4th Latin American Symposium on Theoretical Informatics, pages 88-94, London, UK, 2000. SpringerVerlag.

[4] A. Bernstein and D. Karger. A nearly optimal oracle for avoiding failed vertices and edges. In STOC '09: Proceedings of the 41st annual ACM symposium on Theory of computing, pages 101-110, New York, NY, USA, 2009. ACM.

[5] S. Chechik, M. Langberg, D. Peleg, and L. Roditty. Fault-tolerant spanners for general graphs. In STOC '09: Proceedings of the 41st annual ACM symposium on Theory of computing, pages 435-444, New York, NY, USA, 2009. ACM.

[6] C. Demetrescu and G. F. Italiano. A new approach to dynamic all pairs shortest paths. J. ACM, 51(6):968-992, 2004.

[7] C. Demetrescu, M. Thorup, R. A. Chowdhury, and V. Ramachandran. Oracles for distances avoiding a failed node or link. SIAM J. Comput., 37(5):1299-1318, 2008.

[8] R. Duan and S. Pettie. Dual-failure distance and connectivity oracles. In SODA '09: Proceedings of the Nineteenth Annual ACM -SIAM Symposium on Discrete Algorithms, pages 506-515, Philadelphia, PA, USA, 2009. Society for Industrial and Applied Mathematics.

[9] J. Hershberger and S. Suri. Vickrey prices and shortest paths: What is an edge worth? In FOCS '01: Proceedings of the 42nd IEEE symposium on Foundations of Computer Science, page 252, Washington, DC, USA, 2001. IEEE Computer Society.

[10] N. Khanna and S. Baswana. Approximate shortest paths avoiding a failed vertex : optimal data structures for unweighted graphs. http://www.cse.iitk.ac.in/ sbaswana/publications/algorithmica-09.pdf.

[11] E. Nardelli, G. Proietti, and P. Widmayer. Finding the most vital node of a shortest path. Theor. Comput. Sci., 296(1):167-177, 2003.

[12] L. Roditty. On the k-simple shortest paths problem in weighted directed graphs. In SODA '0\%: Proceedings of the eighteenth annual ACM-SIAM symposium on Discrete algorithms, pages 920-928, Philadelphia, PA, USA, 2007. Society for Industrial and Applied Mathematics.

[13] M. Thorup and U. Zwick. Approximate distance oracles. J. ACM, 52(1):1-24, 2005. 